\title{
195. Über die Spaltung der Doppelbindung im Neoergosterol
} von W. E. Bachmann $\dagger$, M. P. Gava und André S. Dreiding

(9. VII. 59)

Da die Entfernung der Seitenkette in Neocrgosterol (I) zu potentiell interessanten $a r$, B-Steroiden ${ }^{1}$ ) führen könnte, habcn wir Versuche in dieser Richtung unternommen. Schon bei der Strukturaufklärung des Neoergosterols hat H. H. INHOFFEN ${ }^{2}$ ) dic Doppelbindung der Seitenkette mit Ozon gespalten und 2,3-Dimethylbutanal vorgefunden. Bei der gleichen Reaktion hat R. P. JACOBSON ${ }^{3}$ ) das tetracyclische Spaltstück als 3 $\beta$-Acetoxy-ar,B-bisnorcholansäure (IIb) in 9\% Ausbeute isoliert.<smiles>CC(=O)OC1CCc2c(ccc3c2CCC2C3CCC2C(C)/C=C\C(C)C(C)C)C1</smiles><smiles>[R20]C1CCc2c(ccc3c2CC[C@]2(C)[C@H]3CC[C@@H]2C(C)C(=O)O)C1</smiles><smiles>CC(C)OC1CCc2c(ccc3c2CCC2C3CCC2C(C)C(O)C(O)C(I)C(C)C)C1</smiles><smiles></smiles><smiles>[R20]C1CCc2c(ccc3c2CC[C@@]2(C)[C@H]3CC[C@@H]2[C@@H](C)C(OC)OC)C1</smiles>

a: $\mathrm{R}=\mathrm{H}$

$\mathrm{b}: \mathrm{R}=\mathrm{Ac}$<smiles>CC(=O)OC1CCc2c(ccc3c2CCC2(CCCC2=C(C)C=O)C3)C1</smiles>

1) Wir gebrauchen hier die früher von uns (A. S. D.) vorgeschlagene vereinfachte Benennung cier partiell aromatischen Steroide, welche von H. H. INHorfen und Mitarbeitern, Chem. Ber. 87, 1154 (1954), beschrieben worden ist.

2) H. H. INhoffen, Ijicbigs Ann. Chem. 497, 130 (1932). 
Dieselbe Säure wurde von JACOBSON unerwarteterweise auch bei der PerjodsäureSpaltung des mittels Osmiumtetroxyd hergestellten rohen Glycols IIIb in 55-proz. Ausbeute erhalten. Die Bildung der Säure II b anstatt des erwarteten Aldehyds IVb wurde einer Oxydation unter der katalytischen Wirkung der kolloidalen Osmiumverbindungen im rohen Glycol zugeschrieben ${ }^{3}$ ). Dic Säure IIb konnte für den weiteren Abbau der Seitenkette nicht gebraucht werden ${ }^{3}$ ).

Durch eine nochmalige Untersuchung dieser Spaltungsreaktionen ist es uns gelungen, den erwarteten Aldehyd, 3 $\beta$-Hydroxy-ar,B-bisnorcholanaldehyd (IVa) in Form von Derivaten herzustellen.

Bei der Ozonisierung von Neoergosteryl-acetat ( $\mathrm{Ib}$ ) unter Bedingungen, welche die Zurückgewinnung der Hälfte des Eduktes zuliessen, wurden $60 \%$ des gespaltenen Materials als Semicarbazon des Aldehyds IVb gefasst. Mit einem Überschuss an Ozon konnte überhaupt kein kristallisiertes Produkt isoliert werden. Es ist möglich, dass das überschüssige Ozon auch mit dem Benzolring reagiert.

Die Osmiumtetroxyd-Perjodat-Methode führte zum gewünschten Ziel. Ein Osmium-freies Präparat des rohen Glycols IIIa konnte in quantitativer Ausbeute crhalten werden, wenn das Osmiat mit Formaldehyd in alkalischer Lösung reduziert wurde. Durch chromatographische Reinigung wurde eine der zwei möglichen stereoisomeren threo-Formen des Glycols in kristallinem Zustand gefasst. Die Spaltung des rohen Glycolgemisches mit Perjodsäure in Methanol lieferte in 90-proz. Ausbeute direkt das Dimethylacetal des 3 $\beta$-Hydroxy-ar, B-bisnorcholanaldehyds (Va). Offenbar wurde der zuerst gebildete Aldehyd IVa unter den angewandten Reaktionsbedingungen durch das Methanol und die vorhandene Säure acetalisiert. Das Acetal Va wurde schon durch milde Hydrolyse in den amorphen Aldehyd IV a überführt, dessen Infrarotspektrum eine beim Acctal nicht vorhandene Carbonylbande bei $5,84 \mu$ aufwies.

Acetylierung des Acetals Va ergab das kristallisierte Acctoxyacetal Vb, welches bei der Behandlung mit Pyridiniumperbromid-hydrobromid in Essigsäure ${ }^{4}$ ) unter Desacetalisierung 3 $\beta$-Acetoxy-20-bromo-ar, B-bisnorcholanaldehyd (VI) lieferte. Dieser Bromaldehyd spaltete bei der Behandlung mit 2,4-Dinitrophenylhydrazin in saurer Lösung $\mathrm{HBr} \mathrm{ab}^{5}$ ), wobei ein rotes 2,4-Dinitrophenylhydrazon entstand, dem die Struktur eines Derivates des ungesättigten Aldehyds VII zugeschrieben werden kann.

\section{Experimenteller 'Teil ${ }^{6}$ )}

Neoevgosterol und sein Acetat. Neoergosterol, Smp. 150-152 ${ }^{\circ}$, wurde durch Pyrolyse im Hochvakuum von Bisergostatrienol ${ }^{7}$ ) in 23-proz. Ausbeute erhalten. Für den Erfolg dieser Pyrolyse war es notwendig, frisch hergestelltes Bisergostatrienol zu gebrauchen, da die Ausbeute und Reinheit des Produktes mit fortschreitendem Alter des Eduktes abnahm. - Neoergosterol wurde durch Behandlung mit Acetanhydrid und Pyridin bei Zimmertemperatur in das Acetat überführt. Nach Umkristallisation aus Chloroform-Methanol schmolz dieses bei $119-121^{\circ}$ (Lit. ${ }^{7}$ ) 116-117 ${ }^{\circ}$.

Ozonisierung von Neoergosterylacetat. Ein 3-proz. Ozonstrom wurde $20 \mathrm{Min}$. durch eine bei $0^{\circ}$ gehaltene Lösung von $193 \mathrm{mg}$ Neoergosterylacetat (I b) in $10 \mathrm{ml}$ Tetrachlorkohlenstoff geleitet.

3) R. P. Jacobson, J. Amer. chem. Soc. 65, 1789 (1943).

4) C. DJerassi \& C. Scholz, J. Amer. chem. Soc. 70, 417 (1948).

5) V. Mattox \& E. C. Kendall, J. Amer. chem. Soc. 70, 882 (1948).

6) Die Smp. sind unkorrigiert mit Ausnahme derjenigen, die mit korr. bezeichnet sind.

7) A. Windaus \& P. Borgeaud, Liebigs Ann. Chem. 460, 235 (1928). 
Nach Zugabe von $10 \mathrm{ml}$ Essigsäure und $0,5 \mathrm{~g}$ Zinkstaub wurde das Gemisch $5 \mathrm{Min}$. auf dem Wasserbad erhitzt. Dic Aufarbeitung in üblicher Weise, inklusive $\mathrm{NaHCO}_{3}$-Extraktion, ergab eine halbfeste neutrale Masse, aus welcher beim Umkristallisieren aus Mcthanol $90 \mathrm{mg}(47 \%)$ Neoergosterylacetat (Ib), Smp. 116-118 ${ }^{\circ}$, zurückgewonnen wurden. Das in der Mutterlauge enthaltene nicht kristallisierbare Öl wurde mit $150 \mathrm{mg}$ Semicarbazid-hydrochlorid und $200 \mathrm{mg}$ Natriumacetat in $10 \mathrm{ml}$ Methanol in das Semicarbazon von 3 $\beta$-Acetoxy-ar, B-bisnorcholanaldehyd (IVb) überführt. Nach dem Unkristallisieren aus wässerigem Methanol wog es $60 \mathrm{mg}(60 \%$ Ausbeute, bezogen auf verbrauchtes Edukt) und schmolz bei $205^{\circ}$.

$$
\mathrm{C}_{24} \mathrm{H}_{33} \mathrm{O}_{3} \mathrm{~N}_{3} \text { (411) Ber. C 70,0 H 8,09\% Gef. C 70,4 H 8,32\% }
$$

Wenn der Ozonstrom unter den gleichen Bedingungen während 40 Min. durchgeleitet wurde, konnte weder Neocrgosterylacetat (Ib) zurückgewonnen noch kristallisiertes Semicarbazon erhalten werden.

Threo-22,23-Dihydroxy-22,23-dihydro-neoergosterol (II I a). Eine Lösung von $3 \mathrm{~g}$ Neoergosterylacetat (I b) und $2 \mathrm{~g}$ Osmiumtetroxyd in $150 \mathrm{ml}$ trockenem Äther wurde 3 Tagc bei $20^{\circ}$ stehengelassen. Das bcim Abblasen des $\Lambda$ thers zurückgebliebene schwarze Osmiat wurde durch 18 stündiges Frhitzen mit $12 \mathrm{ml} 37$-proz. Formaldehyd, $20 \mathrm{ml} 2$-n. Natriumhydroxyd und $100 \mathrm{ml} \ddot{A}$ thanol reduziert. Die Suspension wurde mit Essigsäure neutralisiert, im Vakuum vollkommen eingcdampft und der gut pulverisicrte Rückstand mit Benzol kontinuierlich extrahiert. Nach dem Abdampfen des Benzols blicb 2,9 $\mathrm{g}(99 \%)$ des rohen Glycolgemisches III a als fast farblose glasartige Masse zurück.

$250 \mathrm{mg}$ dieses rohen Glycols wurden an $10 \mathrm{~g}$ mit Essigester gewaschenem Aluminiumoxyd chromatographiert. Die Hauptfraktion $(192 \mathrm{mg})$ wurde mit Äther-Methanol (9:1) eluiert und

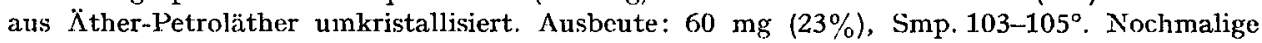

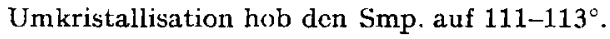

$$
\mathrm{C}_{27} \mathrm{H}_{42} \mathrm{O}_{3}, 1 / 2 \mathrm{H}_{2} \mathrm{O}(423) \quad \text { Ber. C 76,5 } \mathrm{H} \mathrm{10,1 \%} \quad \text { Gef. C 76,2 H 10,1\% }
$$

$3 \beta$-Hydroxy-ar, $B$-bisnorcholanaldehyd-dimethylacetal ( $\mathrm{Va}$ ). Line Lösung von $2,9 \mathrm{~g}$ des rohen Glycols III a und 1,7 g Pcrjodsäure in $50 \mathrm{ml}$ Mcthanol wurde 12 Std. bei Zimmertemperatur stehengelassen. Nach Neutralisicrung mit 1-n. Natronlauge wurde mit $450 \mathrm{ml}$ Wasser verdünnt

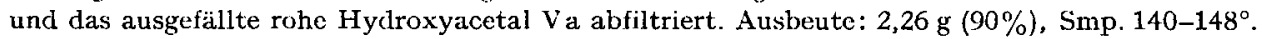
Der Smp. erreichte nach mehrmaligem Umkristallisieren aus Methanol 178,6-180,9 (korr.). Das IR.-Spektrum in $\mathrm{KBr}$ wies keine Absorptionsbande in der Carbonylgegend auf.

$\mathrm{C}_{23} \mathrm{H}_{34} \mathrm{O}_{3}(358) \quad$ Ber. C 77,2 H 9,50 $\mathrm{OCH}_{3} 17,26 \%$ Gef. C 76,7 H 9,84 $\mathrm{OCH}_{3} 16,16 \%$

$3 \beta$-Hydroxy'-ar, B-bisnorcholanaldehyd ( $I V a) .10 \mathrm{mg}$ Hydroxyacetal $\mathrm{Va}$ wurden mit $1 \mathrm{ml}$ Dioxan und 0,5 $\mathrm{ml}$ konzentricrter Salzsäure und $0,5 \mathrm{ml}$ Wasser $5 \mathrm{Min}$. auf $80^{\circ}$ erhitzt. Die übliche Aufarbeitung lieferte den Hydroxy-aldehyd IV a als nicht kristallisierende Masse, dessen IR.Spektrum in $\mathrm{CH}_{2} \mathrm{Cl}_{2}$ eine Carbonylbande bei $5,84 \mu$ zeigte.

2,4-Dinitrophenylhydrazon von IV a: aus Methanol-Essigester gelbe Kristalle. Smp. 230-232 (Zers.).

$$
\mathrm{C}_{27} \mathrm{H}_{32} \mathrm{O}_{5} \mathrm{~N}_{4}(492) \quad \text { Ber. C 65,8 H 6,52 N 11,4\% Gef. C 65,2 H 6,32 N 11,3\% }
$$

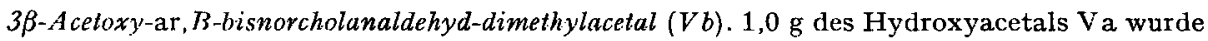
mit $6 \mathrm{ml}$ Acetanhydricl in $6 \mathrm{ml}$ Pyridin $15 \mathrm{Std}$. bei $20^{\circ}$ behandelt. Das überschiussige Acetanhydrid wurde mit $13 \mathrm{ml}$ Wasser zersetzt und die I.ösung mit $10 \mathrm{ml}$ konzentriertcr Salzsäure und viel Eiswasser verdünnt. Das so ausgefällte rohe Acetoxyacetal $\mathrm{Vb}(1,1 \mathrm{~g})$ wurde abfiltriert und aus wässerigem Methanol umkristallisicrt. Smp. $128-130^{\circ}$; Ausbeute $770 \mathrm{mg}(69 \%)$. Ein analytisches Präparat wurde durch nochmaliges Umkristallisieren aus Methanol erhalten. Smp. 134,1$135,4^{\circ}$ (korr.).

$\mathrm{C}_{25} \mathrm{H}_{36} \mathrm{O}_{4}(400) \quad$ Ber. C 75,0 H 9,00 OC. $\mathrm{H}_{3} 15,46 \%$ Gef. C 74,8 H 9,05 $\mathrm{OCH}_{3} 14,67 \%$

Bei der Behandlung von $\mathrm{Vb}$ mit ciner sauren methanolischen Lösung von 2,4-Dinitrophenylhydrazin wurde das Acetal gespalten. Es bildete sich das gelbe 2,4-Dinitrophenylhydrazon des Acetoxyaldchyds IVb in gutcr Ausbeute. Nach Umkristallisation aus Essigester-Benzol schmolzen die kleinen gelben Nadeln bei $234-235^{\circ}$ (Zers.).

$$
\mathrm{C}_{29} \mathrm{H}_{34} \mathrm{O}_{6} \mathrm{~N}_{4}(534) \quad \text { Ber. C } 65,2 \text { H } 6,36 \text { N 10,5\% Gef. C 65,6 H 6,25 N 10,2\% }
$$

38-Acetoxy-20-bromo-ar, B-bisnorcholanaldehyd (VI). Pyridin-hydrobromid-perbromid ( $85 \mathrm{mg})$ wurde ciner warmen $\left(40-50^{\circ}\right)$ Lösung von $100 \mathrm{mg}$ Acetoxyacetal Vb (Smp. 128-130 ${ }^{\circ}$ in $2,7 \mathrm{ml}$ 
Essigsäure zugefügt. Nach einigen Min. war das Perbromid gelöst und hatte unter Entwicklung von $\mathrm{HBr}$ reagiert. Sorgfältiges Verdünnen mit Wasser fällte den Bromaldehyd VI aus, welcher sich nach Kratzen als braune Kristallmasse abfiltrieren liess. Diese wurde aus wässerigem Methanol umkristallisiert, wobei $45 \mathrm{mg}$ weisse Nadeln, Smp. ${ }^{130-132^{\circ}}$, und $42 \mathrm{mg}$ eines unreineren Produktes, Smp. $105-115^{\circ}$, ausfielen (Ausbeute $80 \%$ ). Das analytische Präparat wurde noch aus wässerigem Äthanol umkristallisiert; Smp. 138,4-140 .

$\mathrm{C}_{23} \mathrm{H}_{29} \mathrm{O}_{3} \mathrm{Br}(433) \quad$ Ber. C $63,9 \quad \mathrm{H} 6,77 \quad \mathrm{Br} 18,5 \%$ Gef. C $64,2 \quad \mathrm{H} 7,04 \quad \mathrm{Br} 18,5 \%$

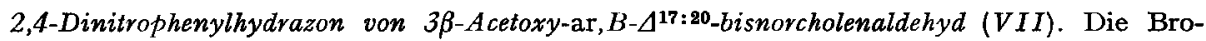
mierung von $100 \mathrm{mg}$ Acetoxyacetal $\mathrm{Vb}$ wurde wie im vorgängig beschricbenen Experiment ausgeführt. Zu der Reaktionslösung wurden direkt 2,5 ml Eisessig, $79 \mathrm{mg}$ Natriumacetat, $54 \mathrm{mg}$ 2,4Dinitrophenylhydrazin und $5 \mathrm{ml}$ Chloroform gegeben. Nach vierstündigem Stehen bei Zimmertemperatur unter Stickstoffatmosphäre wurde das Chloroform abgedampft und durch Wasser ersetzt. Das Produkt wurde mit Benzol ausgeschüttelt und in gewöhnlicher Weise aufgearbeitet. Das Rohprodukt $(140 \mathrm{mg})$ wurde mit $50 \mathrm{ml}$ Tetrachlorkohlenstoff an einer Aluminiumoxydsäule $(1 \times 14 \mathrm{~cm})$ adsorbiert und mit einem 6:4-Tetrachlorkohlenstoff-Chloroform-Gemisch eluiert. Nach Konzentrieren und Kristallisieren aus Essigester-Methanol erhielt man $100 \mathrm{mg}$ (75\%) des 2,4-Dinitrophenylhydrazons von $3 \beta$-Acetoxy-ar,B-17:20-bisnorcholenaldehyd (VII) als orangerote Prismen, Smp. 206-207.

$$
\mathrm{C}_{29} \mathrm{H}_{32} \mathrm{O}_{8} \mathrm{~N}_{4}(532) \quad \text { Ber. C 65,4 H 6,06 } \mathrm{N} \mathrm{10,5 \%} \text { Gef. C 65,0 H 6,34 } \mathrm{N} \mathrm{10,4 \%}
$$

\title{
SUMMARY
}

By a careful sidechain cleavage of neoergosteryl acetate, $3 \beta$-hydroxy-ar,Bbisnorcholanaldehyde could be isolated as its crystalline dimethyl acetal (Va). Acetylation and bromination afforded $3 \beta$-acetoxy-20-bromo-ar, B-bisnorcholanaldehyde (VI), which was converted to the 2,4 -dinitrophenylhydrazone of $3 \beta$-acetoxy-ar, B$\Delta^{17: 20}$-bisnorcholenaldehyde (VII).

\section{Ann Arbor, Michigan, Department of Chemistry, University of Michigan; \\ Zürich, Chemisches Institut der Universität}

\section{Zur Spezifität der modifizierten «KELLER-Reaktion»}

\author{
von H.P. Rieder und M. Böhmer
}

(9. VII. 59)

Eine Reihe von Entdeckungen der letzten Jahre vor allem auf pharmakopsychologischem Gebiet (halluzinogene Wirkung von Lysergsäureverbindungen, von Psilocybin, Dimethyltryptamin, Diäthyltryptamin, Bufotenin, Adrenochrom bzw. Adrenolutin usw.) haben die Indole zu einer biologisch äusserst interessanten Stoffgruppe wcrden lassen. Um so mehr sind heute Methoden $z u$ deren quantitativer Erfassung dringend erwünscht. In einer ersten Mitteilung $z u$ diesem Thema ${ }^{1}$ ) haben wir eine Modifikation der bisher als "KELLER-Reaktion»") bekannten Farbprobe beschrieben, welche als quantitative Indolbestimmung ausgeführt werden kann, und haben darauf hingewiesen, dass der wichtigste Reaktionspartner nicht etwa der Eiscssig, sondern die daraus entstandene Glyoxylsäure ist. Im Prinzip handelt cs sich also um

1) H. P. RIEdER \& M. BöHMER, Experientia 14, 463 (195k).

2) A. Hofmans, Helv. 37, 317 (1954). 